\title{
DOS ESPECIES NUEVAS DE BURSERA (BURSERACEAE) DE LOS ESTADOS DE GUERRERO, MICHOACÁN Y OAXACA (MÉXICO)*
}

\author{
JeRzy Rzedowski y Graciela Calderón de RZEDOWSKI* \\ Instituto de Ecología, A.C., Centro Regional del Bajío, Apdo. postal 386, \\ 61600 Pátzcuaro, Michoacán, México.
}

\section{RESUMEN}

Se describen como nuevas e ilustran Bursera silviae, encontrada en la región costera de Oaxaca, así como B. toledoana, hasta ahora conocida de las porciones inferiores de la Depresión del Balsas pertenecientes a los estados de Guerrero y Michoacán. La primera se relaciona con B. palaciosii Rzed. \& Calderón y con B. staphyleoides McVaugh \& Rzed., distinguiéndose por ser planta intensamente aromática, con flores teñidas de rojo y las masculinas casi siempre trímeras. La segunda se asemeja a B. multifolia (Rose) Engl. y a B. suntui C. A. Toledo, pero difiere en ser arbusto o árbol completamente glabro desde la temprana aparición de las hojas, en sus flores más grandes y las masculinas casi siempre pentámeras.

Palabras clave: Bursera, Burseraceae, México, taxonomía.

\begin{abstract}
Bursera silviae, collected in the coastal area of the state of Oaxaca, and B. toledoana, known from the lower part of the Balsas River Depression in the states of Guerrero and Oaxaca, are described as new and illustrated. The first one is related to B. palaciosii Rzed. \& Calderón and to B. staphyleoides McVaugh \& Rzed., but differs in being a strongly aromatic plant, in its flowers tinged with red and the male ones almost always trimerous. The second is similar to B. multifolia (Rose) Engl. and to B. suntui C. A. Toledo, but distinct in being glabrous from the earliest stage of the leaves and in its larger flowers with the male ones being almost always pentamerous.
\end{abstract}

Kew words: Bursera, Burseraceae, Mexico, taxonomy.

\footnotetext{
* Trabajo realizado con apoyo económico del Instituto de Ecología, A.C. (cuenta 20006), del Consejo Nacional de Ciencia y Tecnología, así como de la Comisión Nacional para el Conocimiento y Uso de la Biodiversidad.
} 
La continuación del estudio sistemático de los cuajiotes y copales ha revelado la existencia de otras dos novedades de la flora mexicana, cuyas descripciones se detallan en seguida.

Bursera silviae Rzed. \& Calderón sp. n.

Arbor vel frutex dioecius ad $7 \mathrm{~m}$ altus; truncus cortice exteriore viridi non vel aliquantum exfolianti, rami juvenes luteoli ad albidi, glabri; folia dispersa vel ad ramulorum brevium apices glomerata, foliolis (1)5-7, 1-4 cm longis, 0.7-2 cm latis, margine integris ad crenatis vel serrulatis; flores solitarii vel saepe in cymis paucifloris dispositi, pedunculis ad 4(6) mm longis; flores masculi 3(4)-meri, feminei 3-meri; calycis lobi triangulares, ca. $0.5 \mathrm{~mm}$ longi, plerumque rubri vel vinacei; petala elliptico-oblonga cucullata ca. $4 \mathrm{~mm}$ longa; ovarium triloculare, stigmata 3 ; pedunculi fructiferi 5-10 $\mathrm{mm}$ longi, fructus triquetri ad globosi, ad $5 \mathrm{~mm}$ longi et lati, maturitate viriduli ad rubelli; pyrenae subglobosae ad aliquantum triquetrae ca. $4 \mathrm{~mm}$ longae et latae, pseudoarillo luteo omnino indutae.

Árbol o a veces arbusto dioico, hasta de $7 \mathrm{~m}$ de alto, con resina aromática; tronco con corteza externa de color verde, nada o poco exfoliante, con látex blanquecino o amarillento, ramas jóvenes amarillentas a blanquecinas, glabras; hojas espaciadas o con tendencia a aglomerarse en los ápices de braquiblastos, pecíolo de (1) 1.5 a 2(2.5) cm de largo, lámina foliar triangular a obovada en contorno general, de 3 a $7 \mathrm{~cm}$ de largo por 3 a $6.5 \mathrm{~cm}$ de ancho, por lo común imparipinnada, foliolos (1) 5 a 7, raquis marginado con un reborde hasta de $0.5 \mathrm{~mm}$ de ancho a cada lado, peciólulo del foliolo terminal hasta de $6 \mathrm{~mm}$ de largo, ensanchándose hacia su extremo distal, los de los foliolos laterales hasta de $1 \mathrm{~mm}$ de largo, a menudo prácticamente ausentes, foliolos ovados, elípticos u oblongos, el terminal con frecuencia romboide y de dimensiones mayores que el resto, de 2.5 a $4 \mathrm{~cm}$ de largo por 1 a $2 \mathrm{~cm}$ de ancho, los laterales de 1 a $3 \mathrm{~cm}$ de largo por 0.7 a $1.2 \mathrm{~cm}$ de ancho, redondeados a agudos en el ápice, en ocasiones ligeramente acuminados, cuneados a redondeados en la base, margen entero a crenado o serrulado, de textura membranácea, nervadura principal evidente por ambas caras, llevando diminutos pelos glandulosos en el pecíolo, en el raquis y en el envés de los foliolos, principalmente en la temprana juventud de las hojas; flores a veces solitarias, más frecuentemente en grupos cimosos de 2 o 3(4), sobre pedúnculos hasta de 4(6) $\mathrm{mm}$ de largo, a menudo provistos de pubescencia de pelos glandulosos cortos, así como de bracteolas por lo común subuladas, de 0.2 a $0.6 \mathrm{~mm}$ de largo, de color de rosa a anaranjadas, a su vez con los márgenes 
ciliado-glandulosos; flores masculinas 3(4)-meras, lóbulos del cáliz triangulares, de ca. $0.5 \mathrm{~mm}$ de largo, por lo común rojos o tendiendo al color guinda, pétalos elíptico-oblongos, cuculados y con el ápice diminutamente encorvado, de ca. $4 \mathrm{~mm}$ de largo y $2 \mathrm{~mm}$ de ancho (extendidos), amarillentos pero a menudo algo manchados de rojo, filamentos desiguales entre sí, de 1.5 a $2 \mathrm{~mm}$ de largo, anteras de unos $2 \mathrm{~mm}$ de largo, gineceo vestigial, prácticamente ausente; flores femeninas trímeras, semejantes a las masculinas, pero con pétalos algo más pequeños (de ca. $3 \mathrm{~mm}$ de largo y extendidos de $1.3 \mathrm{~mm}$ de ancho), anteras de los estaminodios de ca. $0.8 \mathrm{~mm}$ de largo, ovario trilocular, estigmas 3 , de ca. $0.3 \mathrm{~mm}$ de largo por $0.1 \mathrm{~mm}$ de grosor; pedúnculo fructífero de 5 a $10 \mathrm{~mm}$ de largo, por lo común arqueado, frutos solitarios o en conjuntos cimosos de 2 o 3 , aunque con frecuencia aglomerados en mayor número en los extremos de los braquiblastos, triquetros a globosos, trivalvados, hasta de 5 $\mathrm{mm}$ de largo por otro tanto de ancho, a veces apiculados, verdosos a rojizos, glabros, hueso sugbloboso a ligeramente triquetro, de ca. $4 \mathrm{~mm}$ de largo y de ancho, cubierto totalmente por un pseudoarilo amarillo.

Tipo: México, Oaxaca, distrito de Pochutla, municipio de Santa María Huatulco, $500 \mathrm{~m}$ al oeste de la vereda de Aguaje Anona, sitio de muestreo de G. Juárez, 1546'23.5" N, 96¹3'0.5" W, 25.V.2006, G. Juárez García, S. Salas, A. Sánchez, E. Lott y T. Atkinson 2214 (IEB, isotipos por distribuirse).

Material adicional examinado: México, OAXaCa. Distrito de Jamiltepec: 4 km al E de Agua Zarca, municipio de San Pedro Tututepec, R. López G. 677 (IEB). Distrito de Pochutla: $5 \mathrm{~km}$ de la carretera nacional por la brecha a las playas de

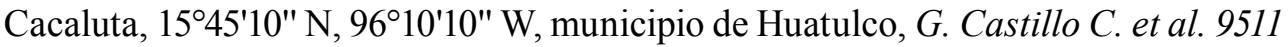
(IEB), 9523 (IEB); km 2 de el camino a Santa Cruz Huatulco, al S de la carretera a Salina Cruz, $R$. Cedillo Trigos y R. Torres 1564 (MEXU); $1 \mathrm{~km}$ al S del rancho del río Zimatán, en el paso hacia Petatengo, municipio de San Miguel del Puerto, M. Elorsa C. 313 (IEB); $1 \mathrm{~km}$ al sur de La Crucecita, Los Tanques, 154' $31.4^{\prime \prime} \mathrm{N}$, 96 $8^{\circ} 13.5^{\prime \prime}$ W, municipio de Santa María Huatulco, M. Elorsa C. 7176 (IEB); $100 \mathrm{~m}$ en línea recta al SW (250 grados) del Aguaje Anona, 1546'23.7" N, 96¹ $13^{\prime} 0.5^{\prime \prime} \mathrm{W}$, municipio de Santa María Huatulco, G. Juárez García et al. 1747 (IEB); 1 km al S del Aguaje Anona, rumbo al río Súchil, 1546'13.3" N, 96¹2'50.5" W, municipio de Santa María Huatulco, G. Juárez García et al. 2365 (IEB); sobre el camino viejo a Santa Cruz Huatulco, entre la estación de campo El Sabanal y el río Xúchitl, 1546'29.1" N, 96²'11.5" W, municipio de Santa María Huatulco, A. Nava Zafra et al. 840 (IEB); en la cima de una loma que está al lado del camino viejo de Santa María Huatul- 
co a Santa Cruz Huatulco, 1545'55.6" N, 9600'29.1" W, municipio de Santa María Huatulco, A. Nava Zafra et al. 1020 (IEB); 15'42.5" N, 96¹'56.8" W, municipio de Santa María Huatulco, S. H. Salas M. et al. 5890 (IEB); estación de campo El Sabanal, 1546'7.8" N, 96¹'40.2" W, municipio de Santa María Huatulco, S. H. Salas M. et al. 5916 (IEB); camino viejo a Santa María Huatulco, 1545'51.9"N, 96¹1'21.7" W, municipio de Santa María Huatulco, S. H. Salas M. et al. 5937 (IEB); Portillo de Zimatán, 1550'32.2" N, 9559'21.7" W, municipio de San Miguel del Puerto, $S$. H. Salas M. et al. 5945 (IEB); $500 \mathrm{~m}$ al sur de la entrada al Sabanal, 1546'3.3" N, 96 $12^{\prime} 1.7^{\prime \prime}$ W, municipio de Santa María Huatulco, A. Sánchez Martínez y A. Nava 48 (IEB); estación El Sabanal, $1450 \mathrm{~m}$ al sur del entronque con la carretera costera No. 200, 1546'8" N, 96¹1'38.4" W, municipio de Santa María Huatulco, A. Sánchez Martínez y A. Nava 101 (IEB); sobre el camino viejo a Santa María Huatulco, 1545'40" N, 96¹1'5.3" W, municipio de Santa María Huatulco, A. Sánchez Martínez y A. Nava 515 (IEB); 450 m después de la estación El Sabanal, 1545'54.8" N, 96¹1'25.6" W, municipio de Santa María Huatulco, A. Sánchez Martínez et al. 574 (IEB); $500 \mathrm{~m}$ al este del río Cacaluta, camino al muestreo 4 de G. Juárez, 1544'38.3" N, 96 10'31.1" W, municipio de Santa María Huatulco, A. Sánchez Martínez et al. 1456 (IEB); este del río Cacaluta, muestreo 4 de G. Juárez, 1544'42.6" N, 96¹0'33" W, municipio de Santa María Huatulco, A. Sánchez Martínez et al. 1474 (IEB). Distrito de Sola de Vega: sitio de muestreo de Gonzalo Juárez en Carrizalillo, municipio de Santiago Teotitlán, 1542'13.2" N, 97¹3'3.7" W, A. Sánchez Martínez et al. 1941 (IEB). Distrito de Tehuantepec: por la carretera costera, $6 \mathrm{~km} \mathrm{~N}$ de Barra de la Cruz, municipio de San Pedro Huamelula, M. Elorsa C. 542 (IEB, MEXU); Barra de la Cruz, desviación, municipio de Santiago Astata, 1550'54" N, 9558'49" W, $M$. Elorsa C. 3184 (IEB); Barra de la Cruz, $3 \mathrm{~km}$ al SW sobre el camino que comunica a la playa Zimatán, $15^{\circ} 49^{\prime} 42^{\prime \prime}$ N, 95 $58^{\prime} 18^{\prime \prime}$ W, municipio de Santiago Astata, $M$. Elorsa C. 3429 (IEB); $300 \mathrm{~m}$ al N de la desviación a Barra de la Cruz, 1550'59" N, 9558'51" W, municipio de Santiago Astata, M. Elorsa C. 6098 (IEB, MEXU); Chacalapa, $2.5 \mathrm{~km}$ de la desviación de la carretera costera, 15 $5^{\circ} 53^{\prime} 36^{\prime \prime} \mathrm{N}, 95^{\circ} 55^{\prime} 42^{\prime \prime}$ W, municipio de San Pedro Huamelula, E. Martínez y M. Elorsa C. 32383 (IEB,

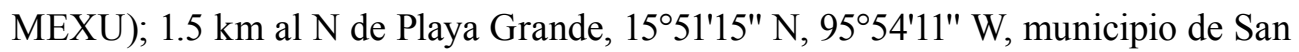
Pedro Huamelula, J. Rivera H. et al. 732 (IEB, MEXU); El Chorro, $110 \mathrm{~m}$ de la entrada, $15^{\circ} 52^{\prime} 29^{\prime \prime}$ N, 960'19" W, municipio de San Pedro Huamelula, S. H. Salas M. 3229 (IEB); carretera a San Isidro Chacalapa, $5 \mathrm{~km}$ al N de la carr. fed., $15^{\circ} 54^{\prime} 9^{\prime \prime}$ N, 9555'42" W, municipio de San Pedro Huamelula, A. Sánchez A. et al. 82 (IEB); $1.6 \mathrm{~km}$ del centro de Barra de la Cruz, rumbo al río Zimatán, por el camino nuevo, 


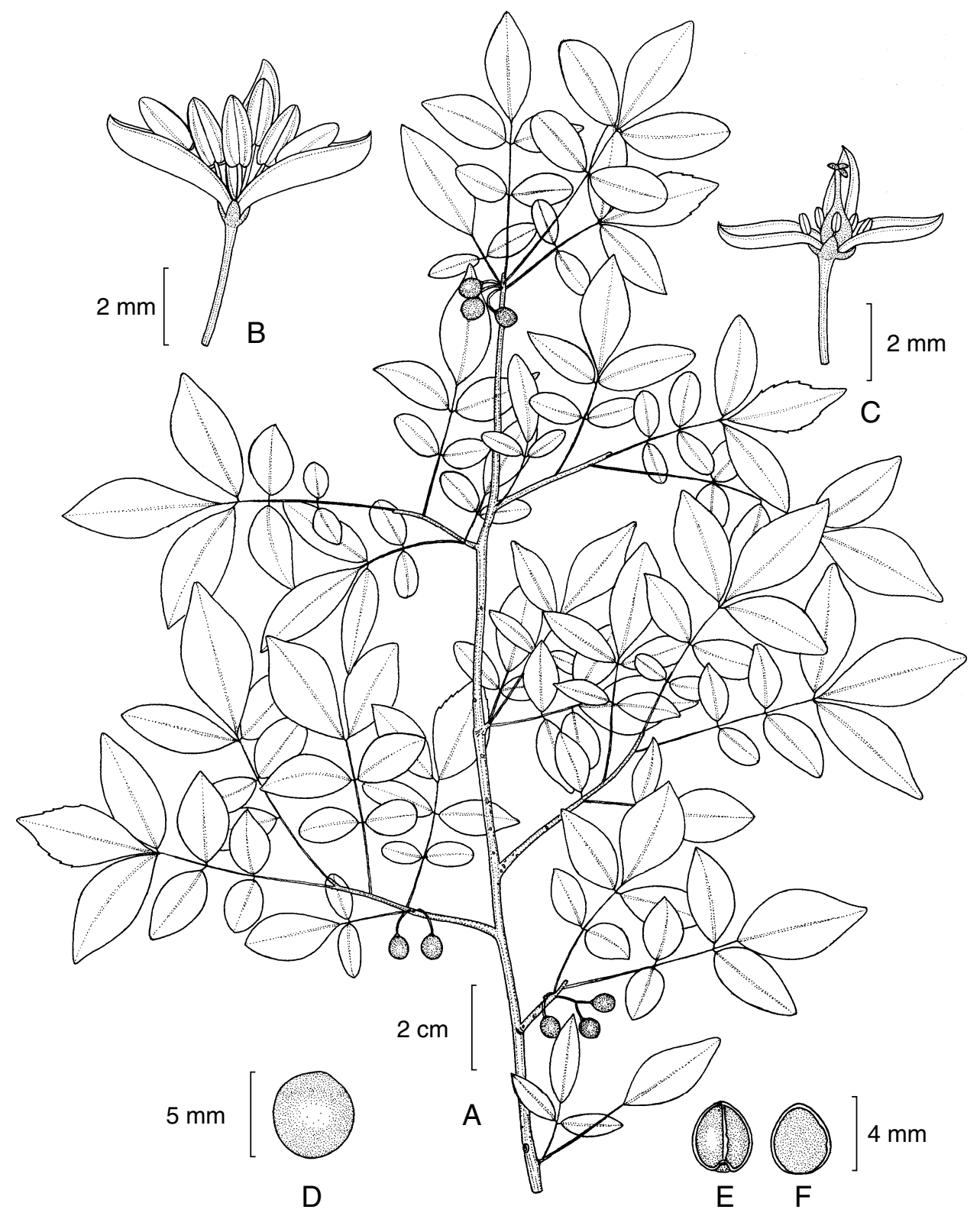

Bursera silviae Rzed. \& Calderón. A. rama con hojas y frutos; B. flor masculina; C. flor femenina; D. fruto; E. y F. hueso visto por ambas caras. Ilustrado por Rogelio Cárdenas. 
$15^{\circ} 49^{\prime} 53^{\prime \prime} \mathrm{N}, 95^{\circ} 58^{\prime} 22.9^{\prime \prime} \mathrm{W}$, municipio de Santiago Astata, A. Saynes V. et al. 3009 (IEB, MEXU).

Bursera silviae constituye un elemento endémico de la región costera de Oaxaca, donde se ha colectado en altitudes entre 0 y $300 \mathrm{~m}$. Se le registraron los nombres comunes de "mulato" y "mulato verde". Florece en abril y mayo y lleva hojas de mayo a octubre.

En virtud de su ovario trilocular, fruto trivalvado, flores mayormente trímeras y catafilos prácticamente ausentes, la nueva especie pertenece a la sección Bursera, y dada la morfología de los foliolos, así como la presencia de látex blanquecino, es afín al grupo que se define alrededor de B. fagaroides (H.B.K.) Engl. y tan es así, que sus primeras colectas fueron identificadas bajo este último nombre.

Dentro del mencionado conjunto $B$. silviae parece estar íntimamente relacionada con $B$. staphyleoides McVaugh \& Rzed., conocida de las partes bajas de la porción michoacana de la Depresión del Balsas, así como con B. palaciosii Rzed. \& Calderón, sólo registrada de la región costera de Jalisco. Las tres especies son glabras o casi glabras, su corteza externa no o apenas se exfolia, las flores y los frutos son manifiestamente pedunculados o pedicelados, los foliolos son relativamente poco numerosos y a menudo de tamaño grande.

De $B$. staphyleoides no se conocen las flores y su principal carácter diferencial consiste en sus hojas trifolioladas, muchas veces también simples. Sus infrutescencias son ramificadas y sus frutos carecen de apículo.

B. palaciosii tiene las hojas con (1)3 o 5 foliolos, las flores y los frutos son solitarios, las flores masculinas son pentámeras, amarillentas o verdosas, los frutos por lo general conservan el estilo en forma de apículo.

B. silviae se caracteriza por hojas con (1)3 a 7 foliolos, las flores de ambos sexos son algo rojizas, casi siempre trímeras, un poco más grandes que las de $B$. palaciosii, y con frecuencia se desarrollan en inflorescencias ramificadas. Sus frutos por lo general carecen de apículos. Se define asimismo como planta intensamente aromática al estrujarse, carácter que no se registra en las otras dos especies, aunque de B. palaciosii se sabe que es muy resinosa.

En algunos municipios del distrito de Tehuantepec $B$. silviae crece en lugares cercanos a los habitados por B. fagaroides. En esa área las dos especies pueden diferenciarse mediante las características resumidas en el Cuadro 1.

El nombre del nuevo taxon se dedica a título de reconocimiento a la M. en C. Silvia H. Salas Morales, destacada bióloga mexicana, a cuya iniciativa, capacidad y entusiasmo se debe la existencia de SERBO (Sociedad para el Estudio de los Re- 
Cuadro 1. Principales diferencias entre Bursera fagaroides y B. silviae.

\begin{tabular}{|l|l|l|}
\hline & \multicolumn{1}{|c|}{ B. fagaroides } & \multicolumn{1}{c|}{ B. silviae } \\
\hline corteza externa del tronco & manifiestamente exfoliante & nada o poco exfoliante \\
\hline ramas jóvenes & grises a cafés & amarillentas o blanquecinas \\
\hline foliolos & hasta de 2(3) cm de largo & hasta de 4 cm de largo \\
\hline flores & $\begin{array}{l}\text { solitarias, sésiles o sobre } \\
\text { pedúnculos hasta de 2 } \mathrm{mm} \\
\text { de largo }\end{array}$ & $\begin{array}{l}\text { solitarias o en grupos } \\
\text { cimosos de 2 a 4, sobre } \\
\text { pedúnculos hasta de } \\
4(6) \text { mm de largo }\end{array}$ \\
\hline $\begin{array}{l}\text { pétalos de las flores } \\
\text { masculinas }\end{array}$ & $(4) 5$ & $3(4)$ \\
\hline frutos & $\begin{array}{l}\text { solitarios, de 4 a } 6 \mathrm{~mm} \mathrm{de} \\
\text { largo }\end{array}$ & $\begin{array}{l}\text { solitarios o en grupos de 2 } \\
\text { o 3, de 3 a 5 mm de largo }\end{array}$ \\
\hline
\end{tabular}

cursos Bióticos de Oaxaca, A.C.), robusta institución mexicana, de gran impacto actual en el contexto de la búsqueda del mejor conocimiento y de la conservación de las plantas de Oaxaca. La Maestra Salas Morales y su grupo de colaboradores han realizado un particular esfuerzo por obtener amplios y completos materiales de herbario de $B$. silviae.

Bursera toledoana Rzed. \& Calderón sp. n.

Frutex vel arbor dioecia ad 6(8) $\mathrm{m}$ alta, glabra; truncus cortice exteriore aliquantum papyraceo exfolianti brunneo; folia saepe ad ramulorum brevium apices glomerata, foliolis 7-11(13), linearibus, lineari-ellipticis vel oblongis, (0.4)1-2(3) cm longis, (1.5)2.5-3.5(4) mm latis, margine integris; flores solitarii vel in paribus dispositi, masculi (4)5(6)-meri, feminei 3(4)-meri; calycis lobi fere liberi, anguste triangulares, ad $1 \mathrm{~mm}$ longi; petala elliptico-oblonga cucullata luteola, 3-5 mm longa; ovarium triloculare, stigmata 3; pedunculi fructiferi ad $1.2 \mathrm{~cm}$ longi; fructus ovoidei aliquantum trigoni, 5.5-7(8) mm longi. trivalvati, maturitate rubelli et saepe albopruinosi; pyrenae triquetrae, $4.5-6 \mathrm{~mm}$ longae, pseudoarillo ut videtur luteo omnino indutae.

Arbusto o árbol dioico hasta de 6(8) $\mathrm{m}$ de alto, glabro; tronco con corteza externa algo papirácea, exfoliante, de color café oscuro, café-amarillento o café-rojizo; ramillas a menudo cubiertas con una delgada capa cerosa de color blanco-grisáceo, 
que es particularmente evidente durante la época seca del año; hojas alternas, a menudo aglomeradas en roseta en los ápices de las ramillas cortas, imparipinnadas, pecíolo algo alado, de 3 a $15 \mathrm{~mm}$ de largo, con frecuencia provisto también de cubierta cerosa, sobre todo hacia la base, con una costilla abultada por el envés, lámina oblonga o elíptica en contorno general, de 3 a 5(7) cm de largo y 1.5 a 3(4) $\mathrm{cm}$ de ancho, raquis ligeramente alado, foliolos 7 a 11(13), opuestos o subopuestos, sésiles o subsésiles, lineares en general o con cierta frecuencia oblongos los más pequeños, o bien, linear-elípticos en particular los apicales, de (0.4)1 a 2(3) $\mathrm{cm}$ de largo y de (1.5)2.5 a 3.5(4) $\mathrm{mm}$ de ancho, redondeados o a veces tendiendo a agudos en el ápice, angostándose levemente hacia la base, margen entero, nervadura principal evidente en ambas caras, las secundarias aproximadamente perpendiculares, mucho menos manifiestas, en ocasiones difíciles de observar, de textura cartácea; flores solitarias o agrupadas por pares, sobre pedúnculos de 2 a $5 \mathrm{~mm}$ de largo y pedicelos de 1 a 4 $\mathrm{mm}$ de largo, bracteolas subuladas o angostamente triangulares, de 0.5 a $1 \mathrm{~mm}$ de largo, a veces ciliadas en el margen; flores masculinas (4)5(6)-meras, lóbulos del cáliz prácticamente separados desde la base, angostamente triangulares, hasta de ca. $1 \mathrm{~mm}$ de largo y $0.5 \mathrm{~mm}$ de ancho, pétalos elíptico-oblongos, de 4 a $5 \mathrm{~mm}$ de largo y 1 a 1.2 (extendidos hasta de 1.6) $\mathrm{mm}$ de ancho, cuculados y con el ápice encorvado, amarillentos, filamentos filiformes, un poco ensanchados en la base, algo desiguales, de 1 a 1.5(2) mm de largo, anteras estrechamente oblongas, de 1.5 a 2 $\mathrm{mm}$ de largo, gineceo vestigial presente; flores femeninas 3(4)-meras, similares a las masculinas, pétalos de $3 \mathrm{a} 4 \mathrm{~mm}$ de largo por 1 (extendidos hasta 2) $\mathrm{mm}$ de ancho, anteras de los estaminodios de ca. $1 \mathrm{~mm}$ de largo, estigmas 3, de aspecto carnoso y granuloso; frutos solitarios o agrupados por varios (hasta 6) a manera de fascículo en los ápices de ramillas cortas, sobre pedúnculos hasta de $1.2 \mathrm{~cm}$ de largo, ovoides y algo trígonos, de 5.5 a 7(8) $\mathrm{mm}$ de largo y 4.5 a $5 \mathrm{~mm}$ de ancho, apiculados, trivalvados, rojizos en la madurez, a menudo cubiertos por una capa cerosa blanquecina; hueso triquetro, de 4.5 a $6 \mathrm{~mm}$ de largo por 4 a $4.5 \mathrm{~mm}$ de ancho, cubierto totalmente por un pseudoarilo aparentemente amarillo.

Tipo: México, Michoacán, alrededores de Zicuirán, municipio de La Huacana, alt. $250 \mathrm{~m}$, bosque tropical caducifolio perturbado, 12.X.1982, J. Rzedowski 37967 (IEB, holotipo; isotipos por distribuirse).

Material adicional examinado: México, Guerrero, $5 \mathrm{~km}$ al $\mathrm{N}$ de Aratichanguio, municipio de Zirándaro, J. L. Contreras y L. Soto 21 (FCME, MEXU); 6 kms al E de Aratichanguio, municipio de Zirándaro, J. Díaz et al. 53 (FCME); 9 


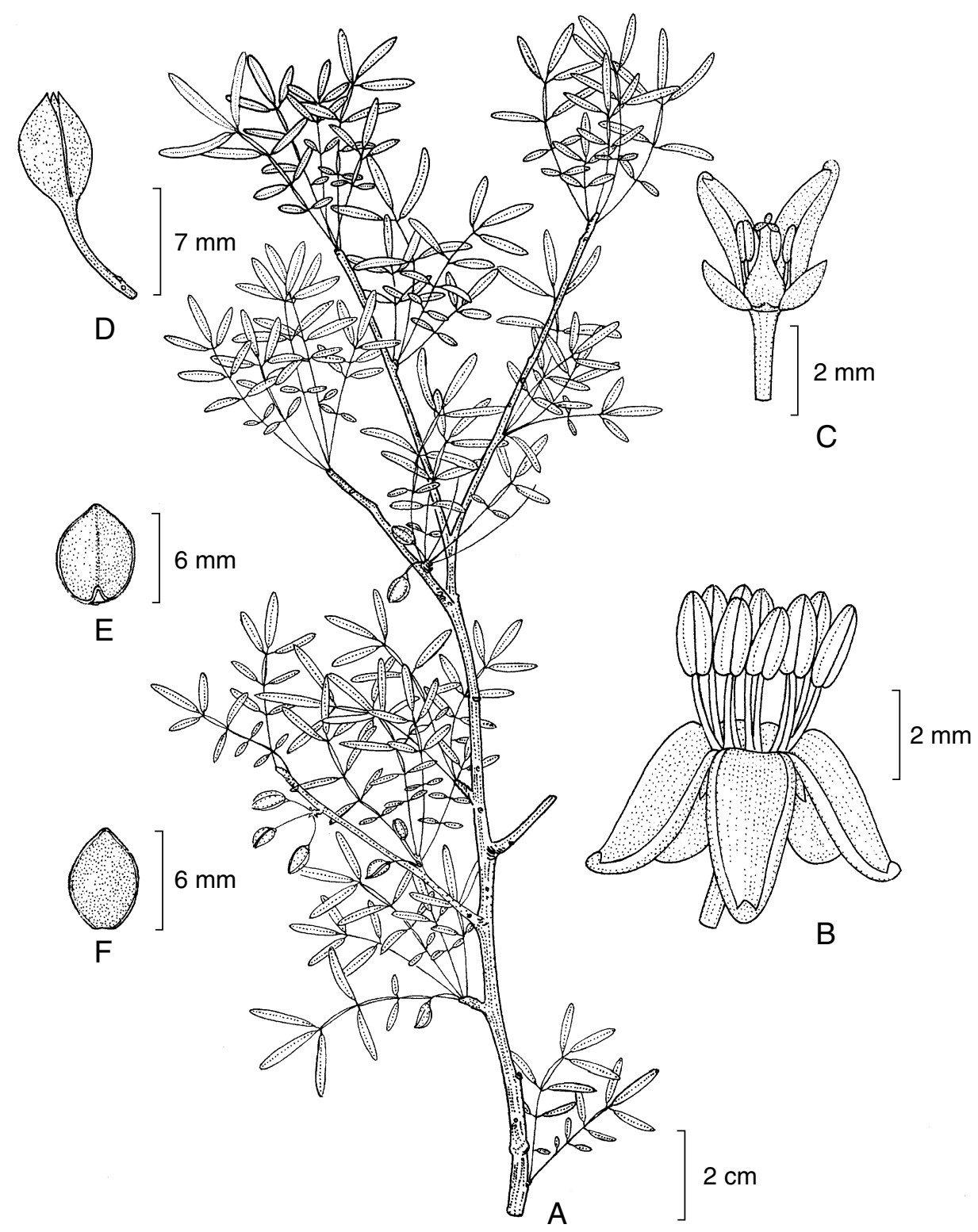

Bursera toledoana Rzed. \& Calderón. A. rama con hojas y frutos; B. flor masculina; C. flor femenina desprovista de un pétalo; D. fruto; E. y F. hueso visto por ambas caras. Ilustrado por Rogelio Cárdenas. 
km adelante de Aratichanguio, hacia Guayameo, S. Torres 1372 (FCME, MEXU). MichOACÁN: $100 \mathrm{~km}$ al NW de Zirándaro, Gro., por la brecha que va a Churumuco, M. Blanco et al. 817 (ENCB, FCME); 2.6 miles N. of Capirio, W. E. Duellman s.n., 14.VIII.1955 (MICH); carretera Uruapan - La Mira, km 258, cerca del poblado Los Olivos, J. D. Hernández B-170 (IEB); a 9 km al W de San Jerónimo, camino a Churumuco, municipio de Huetamo de Núñez, H. E. Martínez y J. C. Soto N. 1441 (ENCB, MEXU); 6 miles NW. of Zicuirán, c. 20 miles SW. of La Huacana, $R$. McVaugh 21951 (ENCB, MEXU, MICH); $4 \frac{1}{2} 2 \mathrm{~km}$ al E de Capirio Nuevo, municipio de Múgica, S. Ontiveros y A. Acosta Fuentes s.n., X.1998 (IEB); alrededores de Zicuirán, municipio de La Huacana, J. Rzedowski 38401 (IEB); camino San Jerónimo - Churumuco, en Esquimucha, municipio de Huetamo, J. C. Soto Núñez y G. Silva R. 3236 (IEB, MEXU); camino San Jerónimo - Churumuco, en San Rafael, municipio de Huetamo, J. C. Soto Núñez y G. Silva R. 3249 (IEB, MEXU); $1 \mathrm{~km}$ al NE de Nuevo Churumuco, carr. a La Huacana, J. C. Soto Núñez 3522 (ENCB); a $1 \mathrm{~km}$ al NE de Churumuco, J. C. Soto Núñez 3522a (MEXU), 3522b (MEXU).

B. toledoana es una especie endémica de las partes más bajas de la porción occidental de la Depresión del Balsas, donde habita en altitudes entre 200 y 400 m, formando parte del bosque tropical caducifolio. Sólo se ha registrado de Michoacán y Guerrero; sin embargo, podría existir también en los sectores adyacentes de Jalisco.

Florece de mediados de mayo a principios de junio. Se encuentra sin follaje entre noviembre y mayo.

Se conoce localmente como "papelillo amarillo" y también con el nombre purépecha de "concanchire"; sus frutos y su resina tienen la reputación de venenosos.

En virtud de su ovario trilocular, fruto trivalvado, tronco con corteza externa exfoliante, así como flores mayormente pentámeras y trímeras, la especie nueva se ubica en la sección Bursera. Sus foliolos lineares a oblongos la colocan en el conjunto que Becerra y Venable (1999) denominan "grupo microphylla", ensamble aparentemente monofilético, en el que los mencionados autores reconocen a $B$. arida (Rose) Standl., B. morelensis Ramírez, B. suntui C. Toledo, B. galeottiana Engl. y B. microphylla A Gray.

En particular, en sus foliolos angostos, relativamente poco numerosos y espaciados, $B$. toledoana se asemeja a $B$. suntui y sobre todo a $B$. multifolia (Rose) Engl., especie que no formó parte del estudio de Becerra y Venable, pero que muy probablemente pertenece al mismo conjunto. Tan es así, que varios ejemplares de 
la planta que aquí se describe se habían identificado con anterioridad como B. multifolia (McVaugh y Rzedowski, 1965, p. 367; Toledo Manzur, 1982, p. 139) o como B. aff. multifolia.

Esta última especie fue descrita del extremo noroeste de Zacatecas y posiblemente existe también en comarcas adyacentes de Jalisco, Durango y Nayarit. Difiere de $B$. toledoana en sus flores más pequeñas (de 2 a $3 \mathrm{~mm}$ de largo), las masculinas trímeras o tetrámeras, en las hojas con foliolos más numerosos ((9)15 a 19) y cortos (hasta de $13 \mathrm{~mm}$ de largo), en la presencia de cortos pelos en las ramillas tiernas y a menudo también en las bases de los peciolos jóvenes, así como en la época de floración (fines de junio y principios de julio).

A su vez, $B$. suntui se conoce solamente del sector oriental de la Depresión del Balsas, en concreto de la parte central y oriental de Guerrero. Esta planta discrepa de $B$. toledoana en la puberulencia presente en ramillas, pecíolos, pedúnculos y pedicelos, en las flores más pequeñas (1.2 a $2 \mathrm{~mm}$ de largo) y más numerosas en las inflorescencias ((1)2 a 6), las masculinas trímeras, en los foliolos más cortos (6.5 a $13.5 \mathrm{~mm}$ ) y relativamente más anchos ( 2 a $4 \mathrm{~mm}$ ), así como en la época de floración (mediados de junio a julio, a veces hasta agosto y septiembre).

El nombre de la planta se dedica como homenaje al Biól. Carlos Arturo Toledo Manzur, quien hace más de 20 años (Toledo Manzur, 1984) reconoció la singularidad de $B$. toledoana, aunque se abstuvo de describirla como especie nueva.

\section{LITERATURA CITADA}

Becerra, J. y D. L. Venable. 1999. Nuclear ribosomal DNA phylogeny and its implications for evolutionary trends in Mexican Bursera (Burseraceae). Amer. Journ. Bot. 86: 1047-1057.

McVaugh, R. y J. Rzedowski. 1965. Synopsis of the genus Bursera L. in western Mexico, with notes on the material of Bursera collected by Sessé \& Mociño. Kew Bull. 18: 317-382.

Toledo Manzur, C. A. 1982. El género Bursera (Burseraceae) en el estado de Guerrero. Tesis. Facultad de Ciencias. Universidad Nacional Autónoma de México. México, D.F. 182 pp.

Toledo Manzur, C. A. 1984. Contribuciones a la flora de Guerrero: Tres especies nuevas del género Bursera (Burseraceae). Biotica 9: 441-449. 
